\title{
QUEEN'S
UNIVERSITY
BELFAST
}

\section{The Inverse Gamma Distribution: A New Shadowing Model}

Yoo, S. K., Cotton, S. L., Zhang, L., \& Sofotasios, P. C. (2021). The Inverse Gamma Distribution: A New Shadowing Model. In 8th Asia-Pacific Conference on Antennas and Propagation August 4 - 7, 2019, Incheon, Korea: Proceedings (Asia-Pacific Conference on Antennas and Propagation (APCAP)). Institute of Electrical and Electronics Engineers Inc.. https://doi.org/10.1109/APCAP47827.2019.9472051

Published in:

8th Asia-Pacific Conference on Antennas and Propagation August 4 - 7 , 2019, Incheon, Korea: Proceedings

Document Version:

Peer reviewed version

Queen's University Belfast - Research Portal:

Link to publication record in Queen's University Belfast Research Portal

Publisher rights

Copyright 2019 IEEE. This work is made available online in accordance with the publisher's policies. Please refer to any applicable terms of use of the publisher.

\section{General rights}

Copyright for the publications made accessible via the Queen's University Belfast Research Portal is retained by the author(s) and / or other copyright owners and it is a condition of accessing these publications that users recognise and abide by the legal requirements associated with these rights.

Take down policy

The Research Portal is Queen's institutional repository that provides access to Queen's research output. Every effort has been made to ensure that content in the Research Portal does not infringe any person's rights, or applicable UK laws. If you discover content in the Research Portal that you believe breaches copyright or violates any law, please contact openaccess@qub.ac.uk. 


\title{
The Inverse Gamma Distribution: A New Shadowing Model
}

\author{
Seong Ki Yoo ${ }^{1}$, Simon L. Cotton ${ }^{1}$, Lei Zhang ${ }^{1}$ and Paschalis C. Sofotasios ${ }^{2,3}$ \\ ${ }^{1}$ Centre for Wireless Innovation, ECIT Institute, Queen's University Belfast, BT3 9DT, UK \\ ${ }^{2}$ Center for Cyber-Physical Systems, Department of Electrical Engineering and Computer Science, \\ Khalifa University, Abu Dhabi 127788, UAE \\ ${ }^{3}$ Department of Electrical Engineering, Tampere University, Tampere 33101, Finland \\ e-mail: \{sk.yoo, simon.cotton, lzhang27\} @qub.ac.uk \& p.sofotasios@ieee.org
}

\begin{abstract}
In this paper, we provide empirical evidence that the inverse gamma distribution is an excellent alternative for the lognormal and gamma distributions which are often used to model shadowing. To illustrate this, we have used field measurements obtained for wearable communication channels. The goodness-of-fit is compared with two other distributions, namely lognormal and gamma. It has been found that the inverse gamma distribution is chosen as the best model in two out of three cases considered in this study.
\end{abstract}

\section{INTRODUCTION}

The lognormal distribution has commonly been used in wireless communications to describe the random fluctuation of the received signal mean level caused by shadowing. Despite its extensive use, one major limitation of using the lognormal distribution is its algebraic representation, which is usually inconvenient for analytic calculations and performance evaluations of communication systems. This presents an even greater challenge when considering composite fading models, which encompass both multipath fading and shadowing.

To overcome the intractability of the lognormal distribution, the gamma distribution has been proposed as an alternative model to describe shadowing [1]. It has been shown that the gamma distribution can provide an adequate description of measurement data previously modeled using the lognormal distribution. Additionally, the use of the gamma distribution has facilitated closed-form expressions for probability density functions (PDFs) of some composite fading models [2], [3]. Unfortunately though, it is not always possible to obtain closed-form solutions. For example, new composite fading models have been proposed using the gamma distribution with the $\kappa-\mu$ and $\eta-\mu$ fading models, respectively [4], [5]; nonetheless, closed-form solutions for their PDFs remain elusive.

Recently, a few studies have proposed the use of the inverse gamma distribution to describe shadowing since it can also exhibit semi heavy-tailed characteristics similar to the gamma distribution [6]. The authors of [6] have shown that the inverse gamma distribution can be used to successfully model the random variation of the mean signal power in composite fading channels. However, to the best of the authors' knowledge, there are no experimental studies which consider the inverse gamma distribution in isolation as a shadowing model. Therefore, for the first time, we empirically validate the inverse gamma distribution for modeling shadowing in this paper.

\section{Shadowing Models}

1) Lognormal Distribution: Let $P$ represent a random fluctuation of the received signal level caused by shadowing. Now considering the case the that $P$ follows a lognormal distribution with location parameter $u$ and scale parameter $\sigma$, the corresponding PDF can be expressed as

$$
f_{P}(p ; u, \sigma)=\frac{1}{p \sigma \sqrt{2 \pi}} \exp \left[-\frac{(\ln (p)-u)^{2}}{2 \sigma^{2}}\right], p>0
$$

where $\ln (\cdot)$ denotes the logarithm to the base $e$.

2) Gamma Distribution: Now considering the case that $P$ follows a gamma distribution with shape parameter $a$ and scale parameter $b$, the corresponding PDF can be obtained as

$$
f_{P}(p ; a, b)=\frac{p^{a-1}}{\Gamma(a) b^{a}} \exp \left(-\frac{p}{b}\right), \quad p>0
$$

where $a>0, b>0$ and $\Gamma(\cdot)$ is the gamma function.

3) Inverse Gamma Distribution: The PDF of the inverse gamma distribution is closely related to the gamma PDF since it follows that $\frac{1}{P} \sim$ inverse $\operatorname{gamma}\left(a, b^{-1}\right)$ when $P \sim$ $\operatorname{gamma}(a, b)$. By letting $S=1 / P, \alpha=a$ and $\beta=b^{-1}$, this can be expressed as follows:

$$
f_{S}(s ; \alpha, \beta)=\frac{\beta^{\alpha}}{\Gamma(\alpha) s^{\alpha+1}} \exp \left(-\frac{\beta}{s}\right), \quad s>0
$$

where $\alpha>0$ and $\beta>0$ are the shape and scale parameters, respectively.

\section{Wearable Channel Measurements}

The wearable channel measurements considered in this study were conducted at $5.8 \mathrm{GHz}$ in the seminar room $(7.92 \mathrm{~m}$ $\times 12.58 \mathrm{~m}$ ) located on the $1^{\text {st }}$ floor of the ECIT Institute at Queen's University Belfast in the UK. The seminar room contained a large number of chairs, desks, a projector and a white board. It also featured an external facing boundary wall constructed entirely from glass with some metallic supporting pillars [Fig. 1(a)]. The seminar room was unoccupied for the duration of the experiments, facilitating pedestrian free wearable channel measurements.

The transmitter (TX) was positioned on the front-central chest region at a height of $1.42 \mathrm{~m}$ of an adult male of height $183 \mathrm{~m}$ and weight $73 \mathrm{~kg}$ [Fig. 1 (b)]. The purposely developed wearable node used for the TX consisted of an ML 5805 and was configured to generate a continuous wave signal with an 
TABLE I

Parameter Estimates for the Lognormal, Gamma and InVErse Gamma Distributions for All of the Wearable Channel MEASUREMENTS ALONG WITH THE NUMERICAL VALUES OF THE CORRESPONDING RAD.

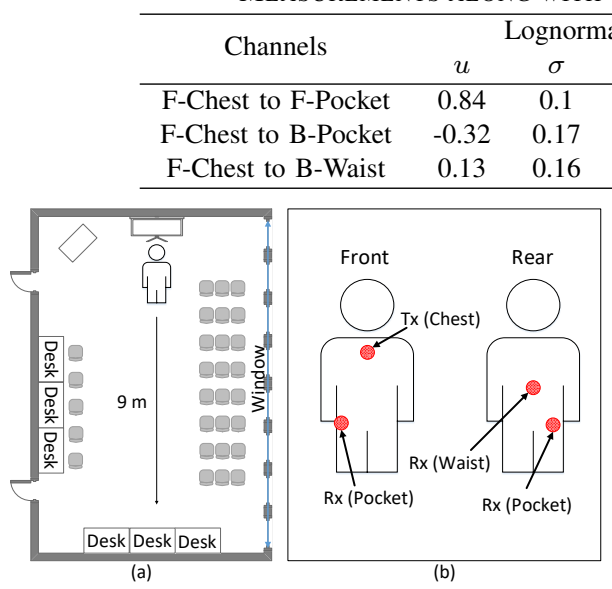

Fig. 1. (a) Wearable measurement environment and (b) the locations of the wireless TX and three different RX nodes.

output power of $+17.6 \mathrm{dBm}$. The receiver $(\mathrm{RX})$ was alternated between three different body locations. These were the rearcentral waist region at a height of $1.15 \mathrm{~m}$ and both right frontand back-pocket of his clothing at a height of $0.92 \mathrm{~m}$. The RX wearable node consisted of an ML5805 transceiver and a PIC32MX which acted as a baseband controller. This configuration allowed the analog received signal strength output by the ML5805 to be sampled with a 10-bit resolution at a sample rate of $1 \mathrm{KHz}$. The antennas used by both the TX and RX were $+2.3 \mathrm{dBi}$ sleeve dipole antennas. During the measurement, the test subject walked along in a straight line within seminar room, covering a total distance of $9 \mathrm{~m}$.

\section{Results}

The shadowing was extracted from the raw measurement data using a moving average of 500 samples. Then, the corresponding parameters for the lognormal and gamma distributions were estimated using maximum likelihood estimation, whereas those for the inverse gamma distribution were obtained using non-linear least squares routine. To provide a quantitative measure of the goodness-of-fit for the lognormal, gamma and inverse gamma distributions with the measurement data, the resistor-average distance (RAD) between empirical and theoretical distributions was calculated [7].

As an example of the results of the model fitting, Fig. 2 shows the received signal power with the superimposed shadowing and the CDFs of the lognormal, gamma and inverse gamma distributions fitted to the measurement data for the front-chest to front-pocket wearable link. As we can see from Fig. 2(b), all three distributions were in excellent agreement with the measurement data. Table I provides the parameter estimates for all of the considered wearable channels to allow the reader to reproduce their own simulated shadowing data. As a further test of the goodness-of-fit, the numerical values of RAD are also given in Table I. It is evident that the inverse gamma distribution provided the smallest RAD for both the front-chest to front-pocket and front-chest to back-pocket links whereas the gamma distribution provided the smallest RAD for the front-chest to back-waist link.

\begin{tabular}{cccccc}
\multicolumn{3}{c}{ Gamma } & \multicolumn{3}{c}{ Inverse Gamma } \\
$a$ & $b$ & RAD & $\alpha$ & $\beta$ & RAD \\
\hline 108.11 & 0.02 & 0.0008 & 109.03 & 250.07 & $\mathbf{0 . 0 0 0 6}$ \\
35.69 & 0.02 & 0.0018 & 38.29 & 27.13 & $\mathbf{0 . 0 0 0 9}$ \\
40.78 & 0.03 & $\mathbf{0 . 0 0 0 6}$ & 40.56 & 46.55 & 0.0020 \\
\hline
\end{tabular}
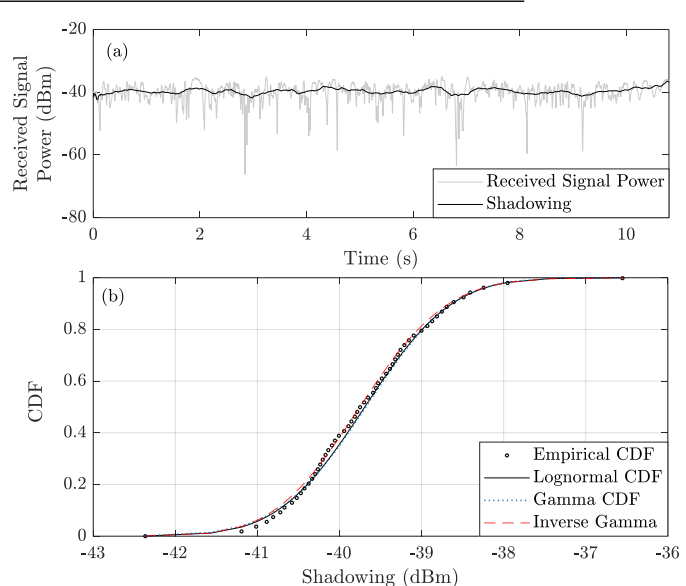

Fig. 2. Received signal power with a superimposed local mean along with the empirical, lognormal, gamma and inverse gamma CDFs for the front-chest to front-pocket wearable link.

\section{CONCLUSION}

In this paper, the inverse gamma distribution has been considered as an alternative distribution to model shadowing. Carrying out channel measurements for wearable communications, which are known to be prone to shadowing, it has been found that the inverse gamma distribution outperformed the lognormal and gamma distributions in two out of three cases considered in this study.

\section{ACKNOWLEDGEMENT}

This work was supported in part by the U.K. Engineering and Physical Sciences Research Council under Grant No. EP/L026074/1, the Department for the Economy Northern Ireland through Grant No. USI080 and also by Khalifa University under Grant No. KU/RC1-C2PS-T2/8474000137 and Grant No. KU/FSU-8474000122.

\section{REFERENCES}

[1] A. Abdi and M. Kaveh, "On the utility of gamma pdf in modeling shadow fading (slow fading)," in Proc. IEEE VTC, vol. 3, May 1999, pp. 2308 2312.

[2] _ "K distribution: An appropriate substitute for Rayleigh-lognormal distribution in fading-shadowing wireless channels," IET Electron. Lett., vol. 34, no. 9, pp. 851-852, Apr. 1998 .

[3] I. Kostić, "Analytical approach to performance analysis for channel subject to shadowing and fading," IEE Proc. Commun., vol. 152, no. 6, pp. 821-827, Dec. 2005.

[4] S. K. Yoo, S. L. Cotton, P. C. Sofotasios, and S. Freear, "Shadowed fading in indoor off-body communication channels: A statistical characterization using the $\kappa-\mu$ / gamma composite fading model," IEEE Trans. Wireless Commun., vol. 15, no. 8, pp. 5231-5244, Aug. 2016.

[5] P. C. Sofotasios and S. Freear, "The $\eta-\mu /$ gamma composite fading model," in Proc. IEEE ICWITS, Oct. 2010, pp. 1-4.

[6] S. K. Yoo et al., "The $\kappa-\mu /$ inverse gamma and $\eta-\mu /$ inverse gamma composite fading models: Fundamental statistics and empirical validation," IEEE Trans. Commun., Dec. 2017.

[7] D. Johnson and S. Sinanovic, Symmetrizing the kullback-leibler distance. Rice University Working Paper, 2001 